\title{
Editorial
}

\section{Suffering from Pain: Reflexology as an Advantageous Adjuvant to Palliative Care}

\author{
Chelli Levi, CR; Michael Silbermann, PhD* \\ The Middle East Cancer Consortium, 45 Yotam Street, Haifa 34675, Israel \\ "Corresponding author \\ Michael Silbermann, PhD \\ The Middle East Cancer Consortium, Israel; E-mail: cancer@mecc-research.com
}

Article information

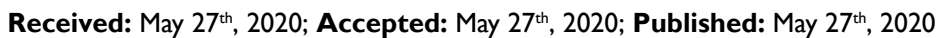

\section{Cite this article}

Levi C, Silbermann M. Suffering from pain: Reflexology as an advantageous adjuvant to palliative care. Palliat Med Hosp Care Open J. $2020 ; 6(\mathrm{I})$ : el-e2. doi: 10.17|40/PMHCOJ-6-e009

$\mathrm{R}$ eflexology is a non-invasive, complementary therapy that is based on the theory that, by applying controlled pressure to certain reflex zones in the palm and foot, channels along the nervous system are stimulated, thereby facilitating energy pathways that may be blocked or congested.

The reflex zone is the region in zone therapy that illustrates its interconnection with all parts of the body. It is hypothesized that there is an invisible pathway which runs along the body vertically, from the top of the head down to the tips of the fingers and toes, while each zone represents a specific area of the body. ${ }^{1,2}$ A recent study in India examined the effectiveness of foot massage on the reduction of pain among cancer patients undergoing palliative care. The findings revealed a highly significant improvement in the degree of their pain as compared with that of control patients. ${ }^{3}$

Reflexology is currently being considered as a complementary therapy, widely used in palliative care to alleviate a problem without necessarily determining its underlying cause in order to improve a patient's emotional, physical, psychological and spiritual health and to upgrade the patient's value of life. ${ }^{4}$

Reflexology does not cause any harmful effects as long as necessary preventions are taken for patients with special medical conditions such as blood clot disorders, cancer and heart failure. Therefore, it is imperative that reflexology practitioners take their clients' overall health condition into consideration. Furthermore, practitioners should ask about and listen to their clients regarding their health status, which may be influenced by a variety of factors such as their psychological and emotional well-being. It is crucial to build a trustful rapport between reflexologist and client, through empathy, leading to a meaningful therapeutic relationship. ${ }^{4}$ Any ongoing reflexology treatment strives to reduce stress levels which, in turn, increases the quality of life as it is one way to interrupt the pattern of repetitive stress stemming from by people's lifestyles.

We believe that the coordination and collaboration between this alternative therapy and mainstream medicine provides a beneficial avenue which may enhance and maintain general physical health via decreasing anxiety and stress. ${ }^{5}$

Since the late 1970's, studies have been conducted to assess the efficacy of behavioral and relaxation approaches to controlling nausea/vomiting, anxiety and other side effects associated with chemotherapy; it has become apparent that reflexology and foot massages can be considered a supportive treatment when used in combination with traditional medical treatments; this approach should be executed by an expert, qualified professional to help cancer patients undergoing chemotherapy to feel better and cope better with their disease. ${ }^{6}$

The modern vision of palliative care involves the management of emotional and spiritual needs of patients and their relatives. This is particularly relevant for developing countries, where extended families are still prevalent, religious values still strong and religious practices still common. ${ }^{7}$ Palliative care in general, and reflexology in particular, indeed represent unique approaches for communities and countries with both high- and low-tech medicine to cope with pain and suffering arising from various illnesses.

We recognize that the presence of reflexology services, regardless of the age of the patient, has the potential to carry intrinsic values to far corners of global health care, including personal dignity, maintaining hope for healing and bearing witness. The latter is the practice of actively listening and not shying away from difficult conversations, bad news or the unknown. It also means sharing personal experiences so that patients realize that they are not alone in this journey. ${ }^{8}$ 


\section{CONFLICT OF INTEREST}

The authors declare that they have no conflicts of interest.

\section{REFERENCES}

1. Embong NH, Soh YC, Ming LC, Wong TW. Revisiting reflexology: Concept, evidence, current practice, and practitioner training. J Tradit Complement Med. 2015; 5(4): 197-206. doi: 10.1016/j. jtcme.2015.08.008

2. Coddington W. Healthista Web site. https://www.healthista. com/. Accessed March 1, 2019.

3. Tamilselvi S, Iyeswariya K. A study to assess the effectiveness of foot massage on reduction of cancer pain among palliative care patients. Int J Res Pharmaceut Sci. 2019; 10(4): 2816-2819. doi: 10.26452/ijrps.v10i4.1555

4. Embong NH, Soh YC, Ming LC, Wong TW. Perspectives on reflexology: A qualitative approach. J Tradit Complement Med. 2016;
7(3): 327-331. doi: 10.1016/j.jtcme.2016.08.008

5. Quinn F, Baxter GD, Hughes CM. Complementary therapies in the management of low back pain: A survey of reflexologists. Complement Ther Med. 2007; 16(1): 9-14. doi: 10.1016/j.ctim.2007.07.004

6. Quattrin R, Zanini A, Buchini S, Turello D, Annunziata MA, Vidotti C, et al. Use of reflexology foot massage to reduce anxiety in hospitalized cancer patients in chemotherapy treatment: Methodology and outcomes. J Nurs Manag. 2006; 14(2): 96-105. doi: 10.1111/j.1365-2934.2006.00557.x

7. Silbermann M, ed. Preface in Palliative Care: Perspectives, Practices and Impact on Quality of Life. A Global View. NY, USA: Nova Biomedical; 2017: 13-20.

8. Weaver M, Berger A. Principles and practices of palliative care across cultures and age groups. In: Silbermann M, ed. Palliative Care: Perspectives, Practices and Impact on Quality of Life. A Global View. NY, USA: Nova Biomedical; 2017: 23-31. 